\title{
Muscarinic Targets for Cognition in Schizophrenia; ADHD Comorbidity; Perinatal Risks, Treatment Resistance and SRIs; and Brain Lesions Presenting as Psychiatric Illness
}

By Eric Hollander, MD

This month's CNS Spectrums examines cholinergic mechanisms of cognitive dysfunction in schizophrenia, how attention-deficit/hyperactivity disorder (ADHD) may complicate the course of other psychiatric illness, whether serotonin reuptake inhibitors (SRIs) may induce spontaneous abortions, treatment-resistant obsessivecompulsive disorder (OCD), and how silent brain lesions can present as psychiatric illness.

Patients with organic lesions in neurologically silent brain areas sometimes present only with psychiatric symptoms such as depression, anxiety disorders, schizophrenia, anorexia nervosa, or cognitive dysfunction. Admoas Bunevicius, $M D$, and colleagues present eight cases of patients with brain lesions, including meningiomas, intracerebral cysts, anaplastic oligodendrogliomas, multiform glioblastomas, and occlusive hydrocephalus, who for a significant period of time were diagnosed and treated for psychiatric disorders (such as Alzheimer's disease, schizoaffective disorder, schizophrenia, depression, and organic emotional lability disorder). When neurologic symptoms developed they underwent neuroimaging studies and organic brain lesions were diagnosed. Further treatment required neurosurgical interventions. These cases show that brain tumors can be neurologically silent for periods of time and manifest as psychiatric disorders. Neuroimaging studies are needed when the presentation or course of illness is unusual, or atypical changes in mental status or neurologic symptoms and signs develop. Missing such an organic lesion can have a huge impact on both the patient and clinician.

Thus, organic lesions can present as functional illness, functional illnesses such as ADHD can complicate the course of other psychiatric disorders, neurocognitive impairment can complicate the course of schizophrenia, and medications for depression can be used in combination for treatment resistance and can cause perinatal complications. The alert physician needs be alert to these complex clinical presentations.

Maternal exposure to post-tricyclic antidepressants during pregnancy might be associated with an increased risk of poor birth, obstetrical, and neonatal outcomes. Among these complications, the occurrence of spontaneous abortions represents one of the most dramatic events for the pregnant woman. Salvatore Gentile, MD, reviewes all primary data studies investigating the rate of spontaneous abortions in women treated with SRls during pregnancy. Information from the reviewed studies are scarce and methodologically inadequate to draw definitive conclusions about the risk of spontaneous abortions associated with serotoninergic antidepressant exposure during early pregnancy. Alternatively, parameters such as teratogenic risk or liability

Dr. Hollander is the editor of this journal, Professor of Psychiatry at the Mount Sinai School of Medicine, and director of the Seaver and New York Autism Center of Excellence in New York City. 
of inducing perinatal complications may be considered in choosing the safest serotoninergic antidepressant for the pregnant woman. The art of psychiatry and medicine is in balancing the known risks and benefits of such treatments to the needs of the individual patient.

Donatella Marazziti, MD, and colleagues explored the possible effectiveness and tolerability of the combination of citalopram + clomipramine in 20 treatment-resistant $O C D$ patients. Approximately $50 \%$ of the patients improved significantly after 1 month and after 1 year of treatment. Although limited by a small sample size and open-label design, this is one of the few studies of the combination of two serotonergic compounds in treatment-resistant $O C D$, and confirms its potential usefulness and tolerability. Of course, controlled research on this association in $O C D$ is recommended. Treatment-resistant $O C D$ remains a critical clinical problem, affecting a population as large as that found in schizophrenia, but, in comparison, it has received scant amount of resources and study. This area too represents and unmet need.

Patients with various psychiatric illnesses may also have unrecognized comorbid ADHD. Russell Barkley, MD, and Thomas E. Brown, MD, note that ADHD symptoms may overlap with other psychiatric disorders, and patients may lack insight into these symptoms. ADHD criteria are limited, since adults may not be able to provide a history of onset of symptoms during childhood, and it may be difficult to confirm that ADHD symptoms are not better accounted for by other comorbid psychiatric conditions. Comorbid ADHD is most prevalent among patients with mood, anxiety, substance use, and impulse-control disorders. ADHD can negatively affect outcomes and compromise treatment compliance. Unrecognized ADHD symptoms may also be mistaken for poor treatment response. ADHD therapy may sometimes improve symptoms of certain psychiatric comorbidities and facilitate a fuller recovery. Alternatively, one might consider ADHD as an associated symptom domain that accompanies various core symptom domains, and shapes the expression, course of illness, and treatment response of the core symptom domain.

Cholinergic activity at muscarinic receptors modulates cognitive functions, which may impair psychosocial rehabilitation in patients with schizophrenia. Angela Sellin, MD, and colleagues reviewed the literature on muscarinic function, cognition, and schizophrenia. Agonist activity at acetylcholine muscarinic type 1 receptors may enhance memory and learning in schizophrenia, whereas the anticholinergic activity of several available antipsychotics may interfere with such functions. Clearly, a major unmet need in the treatment of schizophrenia is targeting executive function deficits that contribute to functional impairment and are not addressed by available antipsychotic agents. CNS 


\section{Neuroscience Education Institute}

\section{On-demand CME is just a few clicks away.}

\section{Members have access to over 100 hours of no-cost credit online.}

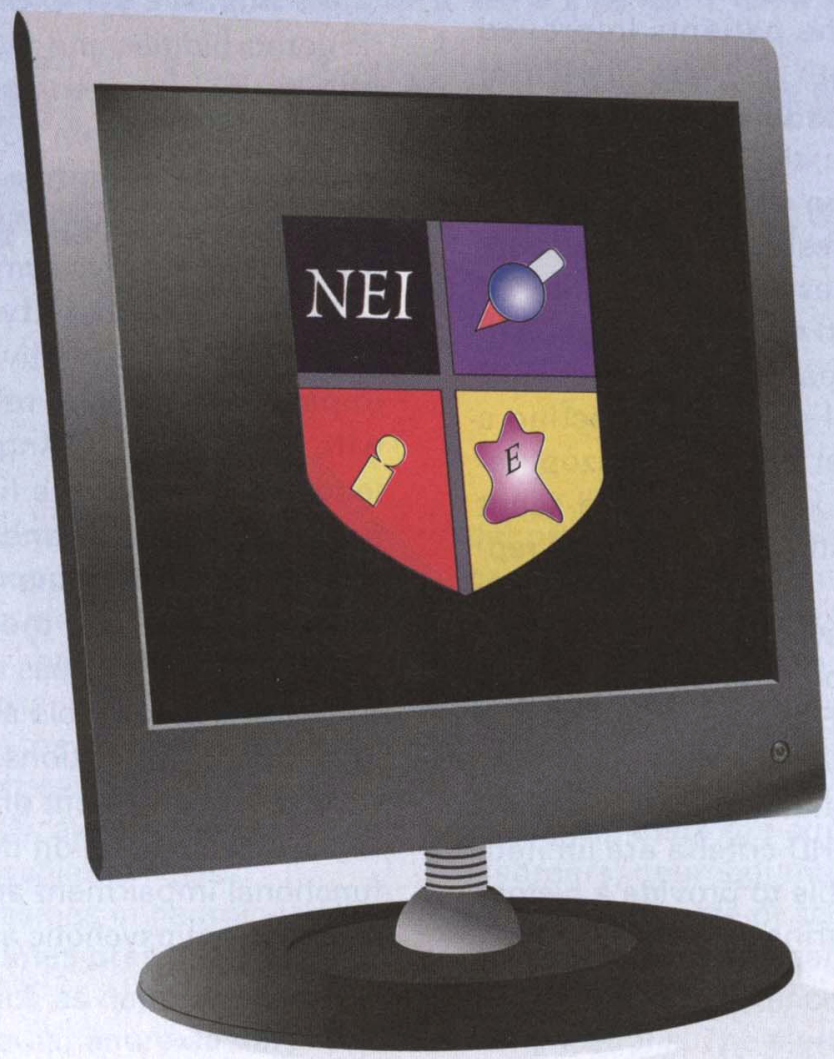

For convenient access to the latest peer-reviewed learning opportunities and AMA PRA Category $1 \mathrm{Credit}^{T M}$

$\mathrm{ADD} / \mathrm{ADHD}$

Alcohol/Substance

Abuse

Anxiety

Bipolar Disorder

CATIE
Children/Adolescents

Dementia

Depression

Fatigue

Metabolic

Mood
Pain

PTSD

Psychosis

Schizophrenia

Sleep

Stress

Find out why we're The Leader in Learning ${ }^{\mathrm{TM}}$ www.neiglobal.com 